Review Article

\title{
Diet and Gut Microbiota in Human Health
}

\author{
R HEMALATHA* \\ Scientist F/Sr. Dy Director, Head, Clinical Division and Microbiology, National Institute of Nutrition, \\ (Indian Council of Medical Research), Hyderabad, India
}

(Received on 28 June 2016; Accepted on 12 July 2016)

\begin{abstract}
There is growing concern and appreciation of the role of our dietary factors in modulating the composition and metabolic activity of the human gut microbiota (microbiome or microflora), which, in turn can impact host health. Beyond prevention and treatment of infections, microbes in the intestine are now known to influence cholesterol metabolism, blood glucose homeostasis, insulin sensitivity, obesity and demonstrate anti-hypertensive and anti-carcinogenic properties, by way of regulating host metabolic pathways and gene expression. Furthermore, recent research advances have expanded our understanding on the importance of the gut microbiome, and its potential involvement in neurological disorders impacting behaviour and mood. Mechanistic insights provide both neurotransmission and immune modulation inflammation routes, through which these effects can be mediated. The expression and function of the key players in adiposity and insulin resistance, such as glucagon-like peptide 1 (GLP-1), free fatty acid receptor 2 (FFAR2), also called G-protein-coupled receptor (GPR41) and peptide YY (PYY) are regulated by microbes and their products. Diet may potentially induce gut bacteria de-regulation and disrupt the basic functions of gut microbiota resulting in low grade inflammation, consequently paving the way for chronic non -communicable diseases (NCDs). In the present review, role of gut microbiome in obesity and diet induced inflammation and their health effects are discussed. Some insight into maternal microbiome in relation to reproductive health and pregnancy outcome, which is an emerging area, is also attempted.
\end{abstract}

Keywords: Gut microbiota; Obesity; Inflammation; Insulin Sensitivity; Maternal Microbiome; Vaginal Microbiome; Probiotics and Prebiotics

\section{Introduction}

The microbiota of the gastrointestinal tract, which is 150 times that of the host genome and 10 times that of host cells, (3.3 million non- redundant bacterial genes) is known to play a crucial role in the physiological and immunological development of the host (Qin et al., 2010). Our gut microbiota contains trillions of microorganisms, belonging to 1000 different species of known bacteria, apart from several unknown microbes. Majorly, the predominant ones are; obligate anaerobes from genera Bacteroides, Eubacterium, Clostridium, Ruminococcus, Peptococcus, Peptostreptococcus, Bifidobacterium, and Fusobacterium, and the less predominant facultative anaerobes are Lactobacillus, Escherichia, Enterobacter, Enterococcus, Klebsiella, and Proteus (Savage, 1977; Manson et

*Authorfor Correspondence: E-mail: rhemalathanin@yahoo.com al., 2008; Eckburg et al., 2005; Martin et al., 2007). Firmicutes is known as the largest bacterial phylum, consisting 200 genera, which includes Lactobacillus, Mycoplasma, Bacillus, and Clostridium (Caricilli and Saad, 2013; Caporaso et al., 2011). Enteroccocus and Escherichia coli constitute less than $1 \%$ of all intestinal microbiota. Anaerobes dominate upon facultative aerobes at the ratio of 1000:1. One third of the gut microbiota is said to be common to most people, while two thirds are unique to each one of us. The 16S rRNA gene sequencing has demonstrated the existence of a large number of yet-unidentified bacteria that inhabit the mammalian intestine (Caricilli and Saad, 2013; Caporaso et al., 2011).

Microbes in the intestine are now known to influence cholesterol metabolism, blood glucose homeostasis, energy harvest, insulin sensitivity and 
blood pressure, apart from controlling gene expression and a number of host metabolic pathways (Claus et al., 2008; Martin et al., 2007; Velagapudi et al., 2010; Wikoff et al., 2009; Li M et al., 2008; Zhao., 2007; Zhao and Shen, 2010). Microbial products such as the short chain fatty acids (SCFA), and conjugated and free fatty acids, are known for their healthpromoting effects (Clemente et al., 2012; Aziz et al., 2013). The general functions carried out by gut microbiota (bacteria) are given in (Fig. 1). Given that $70 \%$ of the immune system is present in the GI tract, the cross-talk between the mucosal immunity and the microbes at the interphase in the intestinal lumen is the determining factor in keeping a delicate balance in the immune system by eliminating invading pathogens, while still maintaining self-tolerance and avoiding auto-immunity and inflammation (Blaise $e t$ al., 2007).

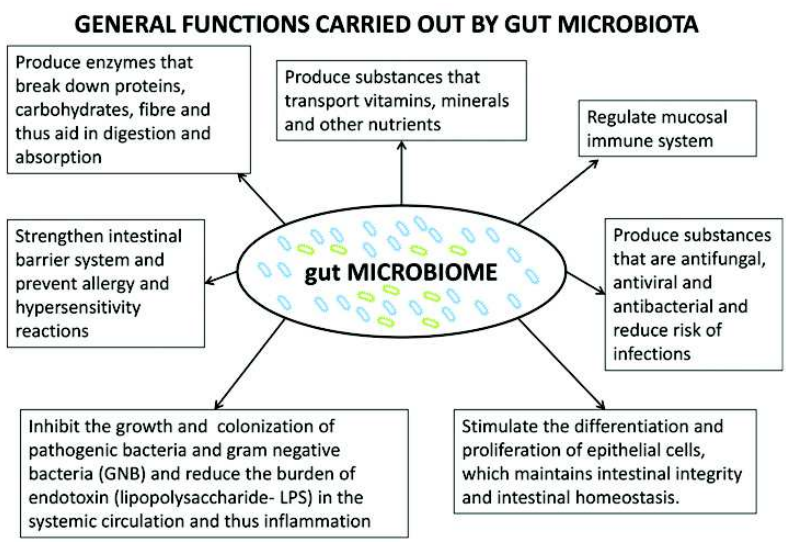

Fig. 1: General functions carried out by gut microbiota

At birth, the intestine is sterile, but intestinal colonisation starts right after birth and evolves as we grow. The newborn's digestive tract is quickly colonised by microorganisms from the mother (vaginal, faecal, skin, breast, etc.). From the third day onwards, the composition of the intestinal flora is dependent on how the infant is fed. While Bifidobacteria dominate the gut microbiome of the breast-fed neonate, babies fed with infant formulas have more gram-negative bacteria in the gut. By the age of three, microbiota becomes stable and similar to that of adults. Gut microbiota's balance can be affected by oral antibiotics, ageing, alcohol, smoking, environmental pollution and diets such as high calorie/ high fat or low micronutrient diets (Michael and Anthony, 2015; Claesson et al., 2011; Makivuokko et al., 2010). Both short- and long-term dietary changes, particularly macronutrients, have a major role in shaping the composition and activity of the intestinal microbiota. Knowledge regarding effects of carbohydrates is relatively better understood; but influence of fats and proteins on the gut microbiota is largely unknown (Michael and Anthony, 2015; Claesson et al., 2011; Makivuokko et al., 2010).

\section{Probiotics and Prebiotics}

With the advances in the knowledge on the important role of dietary components in influencing intestinal bacteria affecting health, the use of functional foods like probiotics and prebiotics have come into trend in the recent past. Probiotic was first conceptualised by a Russian Nobel prize winner and father of modern immunology, Elie Metchnikoff, a scientist at the Pasteur Institute. Elie Metchnikoff (1845-1916) identified an apparent link between consumption of fermented products containing Lactic acid bacteria with human health and longevity (Podolsky, 2012). Probiotic (Greek Language) "for life" was first used by Lilly and Stillwell in 1965 to describe "microorganism that secrete substances, which stimulate the growth of another" (Podolsky, 2012). The FAO/WHO defined probiotics as "live microorganisms which when administered in adequate amounts confer a health benefit on the host"(FAO Report, 2001). They are selected from the strains most beneficial for the host such as Lactobacillus, Bifidobacterium and yeast. Yogurt and milk to which probiotic bacteria have been added, such as acidophilus milk and fermented milk products, such as kefirs, (fermented milk drink containing yeast and beneficial probiotic bacteria) are the primary food sources of probiotics. On the other hand, prebiotics are substances like inulin, oligofructosaccharides (OFS) that are not absorbed through the small intestine and are fermented in the large intestine (gut) by colonic bacteria. Pre-biotics promote the growth of beneficial bacteria in the host (Gibson and Roberfroid, 2004). Pre-biotics are present naturally in fruits, vegetables, onion, garlic, chicory, legumes, nuts, seeds, and most vegetables high in soluble fibre.

\section{Gut Microbiota and Diet-Induced Obesity}

Recent findings show that the gut microbiota has an important role in regulating weight and may be partly 
responsible for the development of obesity (Shen et $a l .$, 2013). Obesity has been linked with reduced bacterial diversity, altered expression of genes and impaired metabolic pathways. The first evidence linking obesity with gut microbiome came from studies on germ- free mice (Backhed et al., 2004; Backhed et al., 2007). Feeding high fat diet to normal mice lead to significantly more weight gain and fat mass and hyperglycemia, than the germ-free mice despite no differences in the diet intake between the two groups (Backhed et al., 2004; Backhed et al., 2007). Likewise, adult germ-free mice colonized with bacteria harvested from the colon of obese mice lead to an increase in body fat within two weeks, suggesting that the energy harvest phenotype could be transmitted by the gut microbiota (Turnbaugh et al., 2006; Turnbaugh et al., 2008). Colonization with obese flora and consequent increase in body fat was associated with decreased expression of fasting-induced adipocyte factor (Fiaf) gene in the ileum of germfree mice (Backhed et al., 2007). Colonization of germ-free mice with specific strains, such as Methanobrevibactersmithii, the methanogenic Archaea species (from the human gut), and Bacteroides thetaiotaomicron also led to body weight gain and increased fat mass (Samuel and Gordon, 2006). However, in experiments on resistin-like molecule- $\beta$ (RELM- $\beta$ ) knockout mouse model, that gains less weight; high-fat diet induced decrease in Bacteriodetes and an increase in both Firmicutes and Proteobacteria. The change in microbiota occurred regardless of whether the mice were obese or not, suggesting that diet was the driving force behind this microbial change (DiBaise et al., 2012; Hildebrandt et al., 2009 ). In contrast, analysis of bacterial $16 \mathrm{~S}$ rRNA, in genetically obese ob/ob mice and their lean counterparts fed the same polysaccharide-rich diet, showed 50\% fewer Bacteroidetes and correspondingly more Firmicutes than their lean litter-mates. This difference was unrelated to differences in food consumption (Ley et al., 2005). Fewer Bacteroidetes and more Firmicutes were also observed in obese humans compared with lean controls (Kotzampassi, 2014).

In humans, gut microbiota during early life has been suggested to predict the subsequent development of overweight and obesity (Kalliomaki et al., 2014; Xiaolin Gao et al., 2015). For instance, greater numbers of Staphylococcus aureus in infancy predicted overweight in children at age seven (Kalliomaki et al., 2014). In contrast, bifidobacterial numbers were higher in children with normal weight (Kalliomaki et al., 2014; Xiaolin Gao et al., 2015). Bifidobacteria produce conjugated linoleic acid, which modulates the fatty acid composition in the liver and adipose tissue in mice (Gorissen et al., 2010). One recent study, found that higher prevalence of the phylum Firmicutes in early infancy was associated with greater weight gain later on (Kotzampassi, 2014). The KOALA cohort study also reported higher Bacteroides fragilis to be associated with greater childhood weight gain (Kalliomaki et al., 2014). Similarly, in overweight pregnant women, Bacteroides and Staphylococcus aureus were relatively higher during pregnancy when compared to normal weight pregnant women (Collado et al., 2008). Since mother's flora influences the microbial flora of the offspring it has been suggested to be one mechanism by which a tendency for obesity is conferred from the parent to the infant. Vaginal delivery seeds the infant's gut with bifidobacteria (Makino et al., 2013). Colonization of bifidobacteria in the infant's gut is further supported by oligosaccharides that are present in the breast milk. Caesarean section delivery or use of antibiotics early in life and type of first feedings are factors that may influence the infants' gut microbiome. Early infant nutrition may thus have life-long consequences through microbial modulation (Makino et al., 2013).

\section{Mechanisms Linking Microbes in Diet Induced Obesity}

The mechanisms implicated in intestinal microbiota and increased storage of calories as fat has been suggested to be due to alterations in the expression of several genes coding for enzymes involved in digestion, absorption and nutrient metabolism (Shen et al., 2013). In addition, short chain fatty acids (SCFAs) are suggested to contribute to up to $10 \%$ of the basal energy requirements of people in developed countries. Paradoxically, this suggests that fibres, which are touted to be associated with weight maintenance, must be contributing to energy by way of SCFA's; especially, when methanogenic Methanobrevibactersmithii species and Bacteroides thetaiotaomicron are abundant in the gut (Samuel and Gordon, 2006). Methanogenic archaea are efficient in fermentation of fibres and 
production of SCFAs that may contribute to more than $10 \%$ of basal energy requirement (Samuel and Gordon, 2006). The mechanistic pathways associating weight maintenance and obesity with microbiome is still at exploratory stage and needs well designed studies to gain more insight into mechanisms. Nevertheless, the suggested mechanisms for microbiota-induced obesity include: (1) fermentation of indigestible polysaccharides and intestinal absorption of monosaccharides and short-chain fatty acids (SCFAs); (2) lipogenesis and glucogeneogenesis in the liver induced by high concentration of SCFAs such as acetate and propionate; (3) upregulation of sterolresponse-element- binding proteins and increased hepatic lipogenesis; (4) suppression of lipoprotein lipase inhibitor, the Fiaf gene; (5) down regulation of AMP-activated protein kinase, that is triggered in response to metabolic stresses such as hypoglycaemia, exercise and hypoxia and (6) suppression of enteroendocrine expression of G-protein-coupled receptor 41 (Gpr41) and peptide PYY (anorexigenic) that inhibit gut motility and increase intestinal transit rate (Velagapudi et al., 2010; Backhed et al., 2004; Shen et al., 2013):

\section{Gut Microbiota, Probiotics and Prebiotics in the Prevention of Obesity}

Microbial population that prevents obesity and that which promotes a healthy state may represent a possible therapeutic strategy in the prevention and management of overweight and obesity (William et al., 2014). Recently, a butyrate-producing species, Faecalibacterium prausnitzii, was negatively associated with obesity and biomarkers of inflammation (Soko et al., 2009). Lin et al. (2012) demonstrated protection from high-fat diet -induced obesity and insulin resistance in mice that were supplemented with acetate, butyrate or propionate. SCFA's modulate gene expression in colonic epithelial cells. For example, butyrate is the energy substrate for colonic epithelium, while acetate and propionate are substrates for lipogenesis and glucogeneogenesis in the liver. SCFA's such as butyrate, may function as microbe-derived signalling molecule that influences carbohydrate and fat metabolism by affecting gene regulation. For instance, butyrate-produced by intestinal bacteria can stimulate glucagon-like peptide 1 (GLP-1) secretion through the free fatty acid receptor 2 (FFAR2), also called G-protein-coupled receptor, in the colonic mucosa. GLP-1 suppresses glucagon secretion, induces glucose-dependent insulin secretion and promotes glucose homeostasis (Lin et al., 2012). The SCFA propionate also modulates energy homeostasis by promoting GPR41 (G proteincoupled receptor 41)-mediated activation of sympathetic neurons (Brown et al., 2003; Kimura et al., 2013). The ability to modulate sympathetic outflow provides another mechanism linking the gut microbiome to the energy expenditure, and metabolic homeostasis. SCFA's are also known to stimulate secretion of peptide YY (PYY), an appetite suppressing peptide (Psichas et al., 2015).

These studies suggest that quality and concentration of SCFA's produced by resident bacteria might prevent obesity. Probiotics such as bifidobacteria regulate gut microbiota, increase portal plasma levels of two gut peptides, GLP-1 and PYY (anorexigenic) and decrease Ghrelin (orexigenic) ( $\mathrm{Li}$ et al., 2011; Parnell et al., 2009). Prebiotics such as fructo-oligosaccharide have been shown to prevent many of the high-fat induced adverse effects including obesity. Apart from promoting the growth of bifidobacteria, bacteria in the gut, prebiotic feeding promotes GLP-1 synthesis in the proximal colon by a mechanism linked to the differentiation of precursor cells into entero-endocrine L-cells (Samuel et al., 2008). All these features are associated with a reduced food intake, decreased body weight gain and fat mass development, and restoration of beta cell mass and glucose-induced insulin secretion (Kobyliak et al., 2016). Prebiotics provide polysaccharides, which are not digestible in our small intestine and therefore make their way to our colon, where they are fermented by a diverse array of microbiota. Fibrerich foods cultivate the various colonic microbiota so that no individual species is dominant. Thus, a dietary pattern rich in prebiotic is one way to promote robust microbial ecosystem that promotes health and prevents obesity and associated complications (Hemalatha et al., 2015).

\section{Genetically Modified Probiotic in the Prevention of Obesity}

Genetic manipulation of known probiotic was investigated recently for prevention of diet induced obesity, with encouraging results. Escherichia coli (strain, Nissle 1917), a probiotic, originally discovered 
by the German military surgeon, Alfred Nissle, during World War I, from the stools of a soldier who did not develop diarrhoea despite a cholera epidemic, is commonly used for treating diarrhoea. This strain (Nissle 1917) has been genetically modified to carry $\mathrm{N}$-acyl-phosphatidylethanolamine (NAPE), a lipid produced in the small intestine. It is converted to $\mathrm{N}$ acylethanolamide (NAE) that can reduce food intake and obesity (Chen et al., 2014). The genetically modified NAPE-producing bacteria could lower food intake and weight gain in mice, in spite of feeding a high-fat diet for 8 weeks (Chen et al., 2014). From all the above listed experimental evidences, it is clear that the high fat diet alters the gut flora, which is linked with metabolic endotoxaemia and associated insulin resistance. Probiotics or prebiotics might help prevent these metabolic changes.

\section{Gut Microbiota Imbalance Implicated in Diet Induced Chronic Low Grade Inflammation}

Low grade chronic inflammatory state and the resultant insulin resistance are known to be the underlying cause of many chronic diseases such as obesity, diabetes, atherosclerosis, cancer and arthritis (Hotamisligil, 2006; Arkan et al., 2005; Wellen and Hotamisligil, 2005). Many age-related diseases such as Parkinson's and Alzheimer's disease are also attributed at least partially to inflammation. Our diet may induce inflammation depending on energy density and micronutrient content (Galland, 2010; Yassour et $a l ., 2016)$. High calorie diet is followed by high postprandial spiking in glucose and lipids (FFA) that generates excess free radicals and stimulates inflammation (Galland, 2010). In addition to the direct effect of free fatty acid (FFA) on inflammatory cascade, a high calorie diet may induce gut bacteria de-regulation, endotoximia and chronic low grade inflammation (Cani, 2007; Cani, 2008).

At the luminal content and host tissues, the intestinal bacteria are in cross-talk with the intestinal epithelium, which acts as the centre-stage integrating pro- and anti-inflammatory signals to regulate host immune responses. Any change in either the quality or quantity of the gut microbes can affect the inflammatory cascade and influence the development of conditions characterized by low-level chronic systemic inflammation, such as obesity and type-2 diabetes (Cani, 2007). A high calorie diet favours reduction in gram -positive bifidobacteria and increase in gram-negative bacteria. Reduction in gram positive bacteria (Bifidobacterium, Lactobacillus, Bacteroides-Prevotella) is associated with down regulation of tight junction (TJ) protein and impairment of intestinal membrane integrity, which becomes more permeable to endotoxin (lipopolysaccharide-LPS) resulting in increasing endotoxin in the blood (Cani, 2007; Cani, 2008). High-fat diet increases gramnegative bacterial (GNB) load in the intestine and excess of LPS released from lysis of GNB results in translocation of LPS into the systemic circulation, wherein it stimulates TLR-4 that triggers chronic low grade inflammation (Cani, 2007). LPS stimulated release of pro-inflammatory cytokines is the key to induce insulin resistance. This was confirmed by continuous sub-cutaneous infusion of LPS that mimics high fat induced changes such as hyperglycemia, hyperinsulinemia, increased fat mass and inflammatory markers and liver triglyceride content (Cani, 2007). Figure 2 depicts impact of high fat/high calorie diet on intestinal bacteria and translocation of LPS into systemic circulation and the genesis of insulin resistance and metabolic syndrome.

A reversal of endotoxaemia with concomitant reduction in systemic inflammation was shown in both high-energy fed and ob/ob mice treated with antibiotic, thereby showing that dietary lipids are not sufficient to induce endotoxemia and systemic inflammation on a background of low number of gram-negative bacteria following antibiotic treatment (Membrez, 2008). Treatment with a prebiotic also had a similar outcome. By enhancing beneficial gram-positive bacteria, prebiotic supplementation prevented body weight gain and fat mass development, reduced endotoxemia, inflammation, oxidative stress, and visceral adiposity. The use of a prebiotic, oligo-fructosaccharide, was shown to lower the adverse effects of high fat diet with selective increase of the gut Bifidobacterium spp. which is reported to reduce intestinal endotoxin levels and improve mucosal barrier function (Roberfroid, 2010). It was demonstrated that the prebiotic modulation of gut microbiota lowers intestinal permeability by a mechanism involving endogenous intestinotrophicproglucagon-derived peptide (GLP-2) production, thereby taming inflammation and metabolic disorders in obesity and diabetes (Cani, 2007; Cani, 2009). Further, it was shown that the GLP-2 antagonist abolished most of the prebiotic effects. 
Pharmacological GLP-2 treatment mimicked prebiotic-induced changes in gut microbiota and decreased gut permeability and inflammation (Cani, 2007; Cani, 2008; Cani, 2009). Put together, these results confirm that the intestinal gram negative bacteria cell wall component- LPS/endotoxin is a prerequisite for chronic low grade systemic inflammation and metabolic disorders and these effects are perhaps mediated through GLP-2.

\section{Bacterial LPS Induced Inflammation May Affect Insulin Signalling and Induce Type 2 Diabetes (T2D)}

In a study, that tested the hypothesis that bacteraemia is an early marker in the development of T2D, phylum proteobacteria was found to be predominant in a cohort of 3280 subjects. Total $16 \mathrm{~S}$ rRNA concentration was higher in those who developed insulin resistance and diabetes 9 years later (Amar et al., 2011). 16S r RNA concentration may be an early biomarker for predicting T2D. Saturated fatty acids, after a high fat/high calorie diet, can directly stimulate TLR molecule. Alternatively, LPS, the outer membrane component of Gram-negative bacteria, may stimulate TLR4 and induce expression of several cytokines, such as TNF- $\alpha$ and IL-6, subsequently resulting in impaired insulin signalling (Fig. 2). Mutation in TLR4 or TLR4 knockout prevents insulin resistance induced by obesity or free fatty acids, suggesting an important

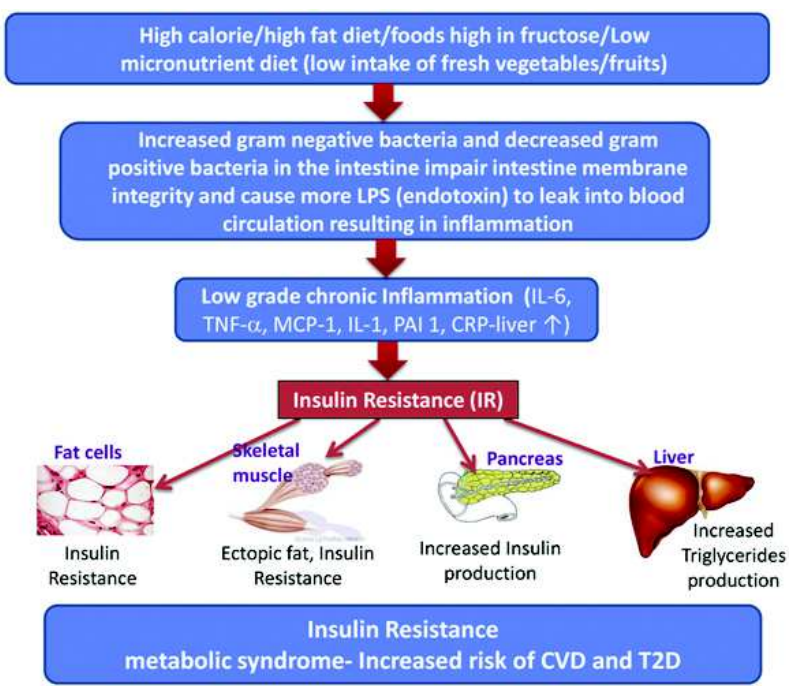

Fig. 2: Impact of high fat/high calorie diet on intestinal microbiota and translocation of LPS into systemic circulation and the genesis of insulin resistance and metabolic syndrome role of TLR4 in the genesis of insulin resistance (Cani, 2007; Cani, 2008). Furthermore, even in preadipocytes, LPS activates TLR4 and increases the expression of several cytokines, TNF- $\alpha$ and IL-6, and impairs insulin signalling in adipocytes. Studies have suggested that bacteria derived LPS can promote the expression of iNOS, NF- $\kappa$ B and activate Mitogen-activated protein kinases (MAPK), all of which interfere with insulin signalling (Rafi et al., 2007). The cumulative effect of LPS-induced release of cytokines (TNF- $\alpha$ and IL6) and nitric oxide may worsen the insulin sensitivity. Added to this, increasing levels of circulating fatty acids may further increase production of nitric oxide and deteriorate insulin sensitivity due to impaired lipoprotein lipase (LPL) activity and increased lipolysis. Dietary supplementation with SCFA (acetate, butyrate or propionate), prebiotic-fructo-oligosaccharide, or probiotic-Bifidobacteria protected against high fat diet-induced obesity and insulin resistance in mice (Lin et al., 2012).

\section{Vaginal Microbiome, Reproductive Health and Pregnancy Outcome}

The human vaginal microbiota, which is predominantly Lactobacillus community, plays an important role in the maintenance of a woman's health. When this predominant Lactobacillus community is disrupted and replaced by anaerobes and gram-negative bacteria, the condition is called bacterial vaginosis (BV) (Hemalatha et al., 2012; 2012). Women with BV have impaired local immunity and increased risk of HIV and other sexually transmitted infections (Fig. 3). BV has been associated with ascending infections and obstetric complications, such as chorioamnionitis, preterm delivery and low birth weight (LBW). In BV the overgrowth of anaerobes produces substances like polyamines and other compounds that increase the vaginal $\mathrm{pH}$ and trigger the release of proinflammatory cytokines IL-1 $\beta$ and IL-8 (Fig. 3). These pro-inflammatory cytokines, IL-1 $\beta$ and IL-8 associated with $\mathrm{BV}$ can profoundly affect reproductive health, reproduction, fertilization, pregnancy and associated complications (Hemalatha et al., 2012; 2012). Exogenous strains of lactobacilli have been suggested as a means of re-instituting a normal healthy vaginal flora (Fig. 3). Carefully selected probiotic strains can eliminate BV associated organisms and restore vaginal health (Mastromarino et al., 2014). Probiotic lactobacilli used in the prevention and 


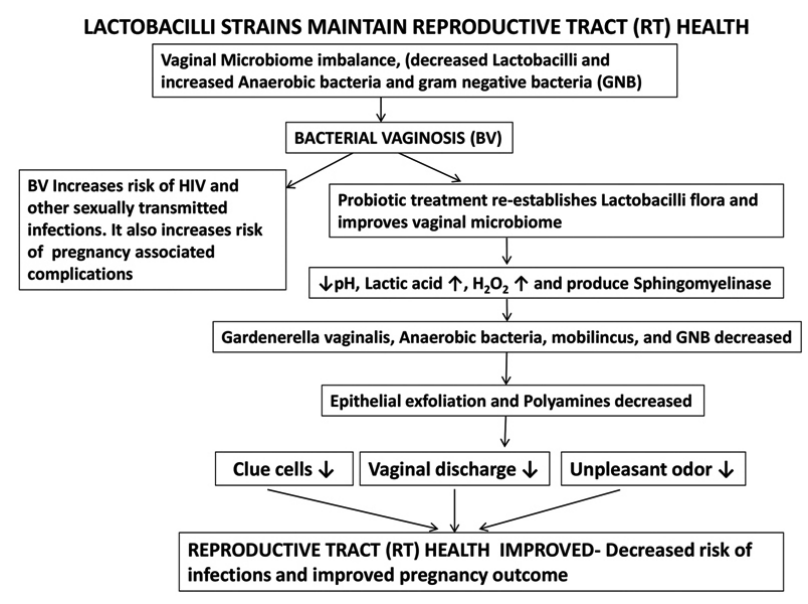

Fig. 3: Mechanism of action of Lactobacilli used in the prevention and treatment of $B V$

treatment of BV exert antibacterial and antiviral effects, restore vaginal $\mathrm{pH}$, ameliorate local inflammation and prevent foetal and neonatal infections (Hemalatha et al., 2012). The administration of beneficial microorganisms such as probiotics either orally or locally (vaginally) are used to restore and maintain a healthy vaginal ecosystem and to maintain reproductive health.

Apart from BV related organisms, disruption of normal vaginal ecosystem and increased vaginal inflammation may also occur with high fat diet. It is seemingly possible that maternal malnutrition and infections may share many of the same adverse consequences mediated through inflammation. In both cases, alterations in physiological functions can be induced and may lead to the development of offspring phenotype that increases the susceptibility to noncommunicable conditions.

\section{Maternal Microbiome and Pregnancy Outcome}

Placenta once considered to be sterile, is actually composed of non-pathogenic commensal microbiota belonging to Firmicutes, Tenericutes, Proteobacteria, Bacteroidetes, and Fusobacteria-phyla (Agaard et al., 2014). Recent research has found the placenta microbiome to be different in term and preterm delivery. A direct correlation of certain bacterial taxa such as paenibacilli was found in term deliveries, while placentas from preterm deliveries had Burkholderia (Aagaard et al., 2014). Likewise, a recent study reported an increase in placenta Fusobacteria and a decrease in Cyanobacteria phyla in low birth weight group, compared with normal birth weight; and a positive correlation between Lactobacillus with increasing birth weight (Zheng et al., 2016). Interestingly, placental microbiome has been shown to be linked with oral microbiome, strengthening the proposed association between maternal dental health and foetal outcomes (Aagaard et al., 2014). An increase in gram-negative bacteria implicated in periodontal disease results in adverse pregnancy outcomes such as the preterm birth and still births (Han et al., 2006; Zi et al., 2015). Even in the absence of periodontal disease, low levels of Lactobacilli in saliva were associated with preterm birth (Durand et al., 2009). Furthermore, every unit increase in $\log$ (10) of Actinomyces naeslundii genospecies 2 (gsp-2), in the oral flora, there was a $60 \mathrm{gm}$ decrease in birth weight and a 0.17 week decrease in gestational age (Dasanayake et al., 2005). In contrast, per unit increase in $\log (10)$ of Lactobacilli case $i$ levels, there was a 42 gm increase in birth weight and a 0.13 week increase in gestational age (Dasanayake et al., 2005). These findings are interesting, in the sense that oral flora can potentially be a good marker for predicting birth outcome.

\section{Other Health Effects of Colonic Bacteria}

There are other less well studied health benefits of colonic bacteria that are not mediated through intestinal integrity, immunity inflammation or energy metabolism. Specific colonic bacteria or probiotic bacteria in fermented milk have been shown to regulate blood lipids, reduce colonic carcinogens and regulate blood pressure.

Renin-Angiotensin-converting enzyme (ACE) system normally maintains blood pressure. ACE inhibitors are commonly used to control hypertension. Supplementation of milk fermented with Lactibacillus helveticus $L B K-16 h$ had demonstrated significant antihypertensive effects in humans. The mechanism behind this may be ACE inhibitor peptides-Ile-ProPro (IPP) and Val-Pro-Pro (VPP) that are produced naturally when milk is fermented with Lactibacillus helveticus and Lactibacillus debrueckii bulgaricus. Large clinical trials are required to confirm this finding. $10-12 \mathrm{mmHg}$ reduction in systolic blood pressure and $5 \mathrm{mmHg}$ reduction in diastolic blood pressure may reduce the risk of stroke by $40 \%$, coronary heart disease by $16 \%$ and all-cause mortality by $13 \%$ (Jakala P and Vapaatalo H, 2010). 
Fermented milk using specific strains of some lactobacilli could lower total cholesterol and lowdensity lipoprotein (LDL) cholesterol and increase high density lipoprotein (HDL). Bile Salt Hydrolase (BHS) present in lactobacilli and bifidobacteria, deconjugate bile acids and increase their rates of excretion (Kumar R et al., 2013). Cholesterol being a precursor of bile acids, is converted to bile acids replacing those lost during excretion. This facilitates reduction in serum cholesterol. Free bile salts are less efficient in the solubilisation and therefore absorption of lipids in the gut is impaired. Cholesterol absorption is reduced in the presence of specific probiotic strains in the intestinal lumen that de-conjugate bile salts (Kumar M, 2012).

Dimethylhydrazin (DMH) is a procarcinogen normally removed by colonic bacteria by adsorption and excretion in faeces. Oxidative radicals and inflammatory molecules such as COX2/PGE2 upregulate colorectal carcinogens and promote formation of carcinogen, azoxymethanemethyldiazoniumion (colorectal cancer) from pro-carcinogens. Beneficial bacteria may reduce the risk of colorectal cancer by promoting excretion of carcinogens, reduction of oxygen radical formation and taming of inflammation. In addition, microbes generate a variety of short chain fatty acids (SCFA's-acetate, butyrate, propionate, valeric, formate) by fermenting dietary carbohydrates (fibre). SCFA's such as acetate, propionate and also butyrate regulate apoptosis and epithelial cell proliferation and reduce the colonic $\mathrm{pH}$ (Liong, 2008). Most importantly, SCFA's stimulate differentiation of colon mucosal cells and impede mucosal atrophy when delivered colorectally or intra-peritoneally in animal models. The low luminal $\mathrm{pH}$ caused by SCFA inhibits

\section{References}

Aagaard K, Ma J, Antony K M, Ganu R, Petrosino J and Versalovic J (2014) The placenta harbors a unique microbiome Sci Transl Med 6 237-265

Amar J, Serino M, Lange C, Chabo C, Iacovoni J, Mondot S et al. (2011) Involvement of tissue bacteria in the onset of diabetes in humans: evidence for a concept Diabetologia 54 3055-61

Arkan M C, Hevener A L, Greten F R, Maeda S, Li Z W, Long J M, Wynshaw-Boris A, Poli G, Olefsky J and Karin M formation of secondary bile acids, which are insoluble and are not taken up by coloncytes and thus lower the carcinogenic potential of secondary bile acids (Liong, 2008). Secondary bile acids are cryptotoxic (toxic to intestinal crypts present in intestinal epithelium) and favour selection of apoptotic resistant cells and promote development of cancer (Kumar et al., 2012).

\section{Conclusion}

In India, a large proportion of population is insulinresistant and the prevalence of diabetes and CHD is high. Accumulating evidence indicates that microbiota of the human intestinal tract plays an important role in health, in particular by mediating many of the effects of diet upon health. Recent research has shown increased proportion of Actinobacteria, Bacteroidetes and gram-negative bacteria and decreased proportion of genus Bifidobacteria with increased serum LPS levels, insulin resistance, increased body weight gain and associated complications such as metabolic syndrome. Elucidating the mechanisms and filling the gaps that exist in the area of diet and gut microbiota are important to develop preventive and therapeutic targeting of the microbiome as an effective strategy for reducing NCDs risk.

Low levels of Lactobacilli in saliva and placenta are associated with preterm and low birth weight babies (LBW). Increasing proportion of oral Actinomyces naeslundiigeno species was also associated with LBW. Studies are needed to unravel the predictive ability of oral microbiota that can be potentially used as a first trimester biomarker to improve maternal health and pregnancy outcomes.

(2005) IKK-beta links inflammation to obesity-induced insulin resistance Nat Med 11 191-198

Aziz Q, Dore J, Emmanuel A, Guarner F and Quigley E M M (2013) Gut microbiota and gastrointestinal health: current concepts and future directions Neuro gastroenterology and Motility 25 4-15

Backhed F, Ding H, Wang T, Hooper L V, Koh G Y, Nagy A, Semenkovich C F and Gordon J I (2004) The gut microbiota as an environmental factor that regulates fat storage Proc Natl Acad Sci U SA 101 15718-15723 
Backhed F, Manchester J K, Semenkovich C F and Gordon J I (2007) Mechanisms underlying the resistance to dietinduced obesity in germ-free mice Proc Natl Acad Sci USA 104 979-98

Blaise H, Rex Gaskins and Annick (2007) Cross-Talk between Probiotic Bacteria and the Host Immune System $J$ Nutr 137 781S-790S

Brown A J, Goldsworthy S M, Barnes A A et al. (2003) The Orphan $\mathrm{G}$ protein-coupled receptors GPR41 and GPR43 are activated by propionate and other short chain carboxylic acids J BiolChem 278 11312-11319

Cani P D, Delzenne N M, Amar J and Burcelin R (2008) Role of gut microflora in the development of obesity and insulin resistance following high-fat diet feeding Pathologie Biologie 56 305-309

Cani P D, Possemiers S, Van de Wiele T et al. (2009) Changes in gut microbiota control inflammation in obese mice through a mechanism involving GLP-2- driven improvement of gut permeability Gut 58 1091-1103

Cani P D, Amar J, Iglesias M A, Poggi M et al. (2007)Metabolicendotoxemia initiates obesity and insulin resistance Diabetes 56 1761-1772

Caporaso J G, Lauber C L, Costello E K et al. (2011) Moving pictures of the human microbiome Genome Biol 12 R50

Caricilli A M and Saad M J (2013) The role of gut microbiota on insulin resistance Nutrients $\mathbf{5} 829-851$

Chen Z, Guo L, Zhang Y, Walzem R L et al. (2014) Incorporation of therapeutically modified bacteria into gut microbiota inhibits obesity J Clin Invest 124 3391-3406

Claesson M J, Cusack S, O'Sullivan O et al. (2011) Composition, variability, and temporal stability of the intestinal microbiota of the elderly Proc Natl Acad Sci USA 108 $14586-4591$

Claus S P, Tsang T M, Wang Y et al. (2008) Systemic multicompartmental effects of the gut microbiome on mouse metabolic phenotypes Mol Syst Bil 4219

Clemente J C, Ursell L K, Parfrey L W and Knight R (2012) The impact of the gut microbiota on human health: An integrative view Cell 148 1258-1270

Collado M C, Isolauri E, Laitinen K and Salminen S (2008) Distinct composition of gut microbiota during pregnancy in overweight and normal-weight women Am J Clin Nutr $\mathbf{8 8}$ 894-899

Dasanayake A P, Li Y, Wiener H, Ruby J D and Lee M J (2005) Salivary Actinomycesnaeslundiigeno species 2 and Lactobacillus casei levels predict pregnancy outcomes $J$ Perio dontol 76 171-177
DiBaise J K, Frank D N and Mathur R (2012) Impact of the Gut Microbiota on the Development of Obesity: Current Concepts Am J Gastroenterol Supp 1 22-27

Durand R, Gunselman E L, Hodges J S, Diangelis A J and Michalowicz B S (2009) A pilot study of the association between cariogenic oral bacteria and preterm birth Oral Dis 15 400-406

Eckburg P B, Bik MB, Bernstein C N, Purdom E, Dethlefsen L, Sargent M, Gill S R, Nelson K E and Relman D A (2005) Diversity of the human intestinal microbial flora Science 308 1635-1638

FAO/WHO Working Group (2002) Report on Drafting Guidelines for the Evaluation of Probiotics in Food London, Ontario, Canada

Galland L (2010) Diet and inflammation Nutr Clin Pract 25 634640

Gao X, Jia R, Xie L, Kuang L, Feng L and Wan C (2015) Obesity in school-aged children and its correlation with gut E.coli and Bifidobacteria: a case-control study BMC Pediatr 15 64

Gibson G R, Probert H M, Loo J V, Rastall R A and Roberfroid M B (2004) Dietary modulation of the human colonic microbiota: updating the concept of prebiotics Nutr Res Rev 17 259-75

Gorissen L, Raes K, Weckx S, Dannenberger D, Leroy F, De Vuyst L and DeSmet S (2010) Production of conjugated linoleic acid and conjugated linolenic acid isomers by Bifidobacterium species Appl Microbiol Biotechnol 87 2257-2266

Han Y W, Fardini Y, Chen C, Iacampo K G, Peraino V A, Shamonki J M and Redline R W (2010) Term stillbirth caused by oral Fusobacteriumnucleatum Obstet Gynecol 115 442-445

Han Y W, Ikegami A, Bissada N F, Herbst M, Redline R W and Ashmead G G (2006) Transmission of an uncultivated Bergeyella strain from the oral cavity to amniotic fluid in a case of preterm birth J Clin Microbiol 44 1475-1483

Hemalatha R, Mastromarino P, Ramalaxmi B A, Balakrishna N V and Sesikeran B (2012) Effectiveness of vaginal tablets containing lactobacilli versus $\mathrm{pH}$ tablets on vaginal health and inflammatory cytokines: a randomized, double-blind study Eur J ClinMicrobiol Infect Dis 31 3097-3105

Hemalatha R, Ramalaxmi B A, KrishnaSwetha G, Kumar P U, Rao D M, Balakrishna N and Annapurna V (2012) Cervicovaginal inflammatory cytokines and sphingomyelinase in women with and without bacterial vaginosis Am J Med Sci 344 35-39

Hemalatha R, Kumar M, Das N, Kumar S N, Challa H R and 
Nagpal R (2015) Effect of Probiotic Lactobacillus salivarius UBL S22 and Prebiotic Fructo-oligosaccharide on Serum Lipids, Inflammatory Markers, Insulin Sensitivity, and Gut Bacteria in Healthy Young Volunteers: A Randomized Controlled Single-Blind Pilot Study J Cardiovasc Pharmacol Ther 20 289-298

Hildebrandt M A, Hoffmann C, Sherrill-Mix S A et al. (2009) High-fat diet determines the composition of the murine gut microbiome independently of obesity Gastroenterology 137 1716-1724

Hotamisligil G S (2006) Inflammation and metabolic disorders Nature 444 860-867

Jakala P and Vapaatalo H (2010) Antihypertensive Peptides from Milk Proteins Pharmaceuticals (Basel) 3 251-272

Kalliomaki M, Collado M C, Salminen S and Isolauri E (2014) Early differences in fecalmicrobiota composition in children may predict overweight Am J Clin Nutr 87 534-538

Kimura I, Ozawa K, Inoue D et al. (2013) The gut microbiota suppresses insulin-mediated fat accumulation via the shortchain fatty acid receptor GPR43 Nat Commun 41829

Kobyliak N, Conte C, Cammarota G et al. (2016) Probiotics in prevention and treatment of obesity: A critical view Nutr Metab (Lond) $\mathbf{1 3} 14$

Kotzampassi K, Giamarellos-Bourboulis E J and Stavrou G (2014) Obesity as a consequence of gut bacteria and diet interactions ISRN Obes $\mathbf{6 5} 1895$

Kumar M, Nagpal R, Kumar R, Hemalatha R, Verma V, Kumar A, Chakraborty C, Singh B, Marotta F, Jain S and Yadav H (2012) Cholesterol Lowering Probiotics as Potential Biotherapeutics for Metabolic Diseases Experimental Diabetes Research ISSN 1560-4284

Kumar M, Nagpal R, Verma V, Kumar A, Kaur N, Hemalatha R, Gautam S and Singh B (2012) Probiotic metabolites as epigenetic targets in the prevention of colon cancer Nutrition reviews 71 23-34

Kumar R, Hemalatha R, Manoj Kumar, Sudarshan Reddy Varikuti, Ramakrishna Athimamula, Ramesh Ramagoni and Narendrababu Kondapalli (2013) Molecular Cloning, Characterization and Heterologous Expression of Bile Salt Hydrolase (Bsh) from Lactobacillus fermentum NCDO394. Mol Bio Report DOI 10.1007/s11033-0132607-2

Ley R E, Backhed F, Turnbaugh P et al. (2005) Obesity alters gut microbial ecology Proc Natl Acad Sci USA 102 1107011075

Li J V, Ashrafian H, Bueter M et al. (2011) Metabolic surgery profoundly influences gut microbial-host metabolic crosstalk Gut 60 1214-1223
Li M, Wang B, Zhang M et al. (2008) Symbiotic gut microbes modulate human metabolic phenotypes Proc Natl Acad Sci USA 105 2117-2122

Lin H V, Frassetto A, Kowalik E J et al. (2012) Butyrate and propionate protect against diet-induced obesity and regulate gut hormones via free fatty acid receptor 3-independent mechanisms PLoS One 7 e 35240

Liong M T (2008) Roles of Probiotics and Prebiotics in Colon Cancer Prevention: Postulated Mechanisms and In-vivo Evidence Int J Mol Sci 9 854-863

Makino H, Kushiro A, Ishikawa E et al. (2013) Mother-to-infant transmission of intestinal bifidobacterialstrains has an impact on the early development of vaginally delivered infant's microbiota PLoS One $\mathbf{8}$ e78331

Makivuokko H, Tiihonen K, Tynkkynen S, Paulin L and Rautonen N (2010) The effect of age and non-steroidal antiinflammatory drugs on human intestinal microbiota composition Br J Nutr 03 227-34

Martin F P, Dumas M E, Wang Y et al. (2007) A top-down systems biology view of microbiome-mammalian metabolic interactions in a mouse model Mol Syst Biol 3112

Manson J M, Rauch M and Gilmore M S (2008) The commensal microbiology of the gastrointestinal tract Adv Exp Med Biol 635 15-28

Mastromarino P, Hemalatha R, Barbonetti A et al. (2014) Biological control of vaginosis to improve reproductive health Indian J Med Res 140 Suppl S91-97

Membrez M, Blancher F, Jaquet M et al. (2008) Gut microbiota modulation with norfloxacin and ampicillin enhances glucose tolerance in mice FASEB J 22 2416-2426

Michael A C and Anthony R B (2015) The Impact of Diet and Lifestyle on Gut Microbiota and Human Health Nutrients 7 17-44; doi: 10.3390/nu7010017

Parnell J A and Reimer R A (2009) Weight loss during oligofructose supplementation is associated with decreased ghrelin and increased peptide YY in overweight and obese adults $A m J$ Clin Nutr 89 1751-1759

Podolsky S H (2012) Metchnikoff and the microbiome Lancet 380 (9856) 1810-1811

Psichas A, Sleeth M L, Murphy K G et al. (2015) The short chain fatty acid propionate stimulates GLP-1 and PYY secretion via free fatty acid receptor 2 in rodents Int $J$ Obes (Lond) 39 424-429

Qin J, Li R, Raes J, Arumugam M, Burgdorf K S, Manichanh C, Nielsen T and Pons N (2010) A human gut microbial gene catalogue established by metagenomic sequencing Nature $46459-65$ 
Rafi M M, Yadav PN and Rossi A O (2007) Glucosamine inhibits LPS-induced COX-2 and iNOS expression in mouse macrophage cells (RAW 264.7) by inhibition of p38-MAP kinase and transcription factor NF-kappaB Mol Nutr Food Res 51 587-593

Roberfroid M, Gibson G R, Hoyles L et al., (2010) Prebiotic effects: metabolic and health benefits Br J Nutr 104 Suppl 2 S1-63

Samuel B S and Gordon J I (2006) A humanized gnotobiotic mouse model of host-archaeal-bacterial mutualism Proc Natl Acad Sci USA 103 10011-10016

Samuel B S, Shaito A, Motoike T et al. (2008) Effects of the gut microbiota on host adiposity are modulated by the shortchain fatty-acid binding $\mathrm{G}$ protein-coupled receptor, $\mathrm{Gpr}$ 41 Proc Natl Acad Sci USA 105 16767-16772

Savage D C (1977) Microbial ecology of the gastrointestinal tract Annu Rev Microbiol 31 107-133

Shen J, Obin M S and Zhao L (2013) The gut microbiota, obesity and insulin resistance Mol Aspects Med 34 39-58

Soko H, Seksik P, Furet J P, Firmesse O, Nion-Larmurier I, Beaugerie L, Cosnes J, Corthier G, Marteau P and Dore J (2009) Low counts of Faecalibacteriumprausnitzii in colitis microbiota Inflamm Bowel Dis 15 1183-1189

Turnbaugh P J, Ley R E, Mahowald M A, Magrini V, Mardis E R and Gordon J I (2006) An obesity-associated gut microbiome with increased capacity for energy harvest Nature 444 1027-1031

Turnbaugh P J, Backhed F, Fulton L and Jeffrey I (2008) Gordon. Marked alterations in the distal gut microbiome linked to diet induced obesity Cell Host Microbe 3 213-223

Turnbaugh P J, Hamady M, Yatsunenko T et al. (2009) A core gut microbiome in obese and lean twins Nature 457 480484
Velagapudi V R, Hezaveh R, Reigstad C S, Gopalacharyulu P, Yetukuri L, Islam S, Felin J, Perkins R, Boren J, Oresic M and Bäckhed F (2010) The gut microbiota modulates host energy and lipid metabolism in mice J Lipid Res 51 11011112

Wellen K E and Hotamisligil G S (2005) Inflammation, stress, and diabetes J Clin Invest 115 1111-1119

Wikoff W R, Anfora A T, Liu J, Schultz P G, Lesley S A, Peters E C and Siuzdak G (2009) Metabolomics analysis reveals large effects of gut microflora on mammalian blood metabolites Proc Natl Acad Sci USA 106 3698-3703

William A Walters A, ZechXu B and Rob Knight (2014) Metaanalyses of human gut microbes associated with obesity and IBD FEBS Letters 588 4223-4233

Yassour M, Lim M Y, Yun H S et al. (2016) Sub-clinical detection of gut microbial biomarkers of obesity and type 2 diabetes Genome Med 817

Zhao L and Shen J (2010) Whole-body systems approaches for gut microbiota-targeted, preventive healthcare J Biotechnol 149 183-190

Zhao L, Kwon M J, Huang S, Lee J Y, Fukase K, Inohara N and Hwang D H (2007) Differential modulation of Nods signaling pathways by fatty acids in human colonic epithelial HCT116 cells J Biol Chem 282 11618-11628

Zheng J, Xiao X, Zhang Q, Yu M, Xu J, Qi C and Wang T (2016) The programming effects of nutrition-induced catch-up growth on gut microbiota and metabolic diseases in adult mice Microbiology open 5 296-306 doi: 10.1002/mbo3.328

Zi M Y, Longo P L, Bueno-Silva B and Mayer M P (2015) Mechanisms Involved in the Association between Periodontitis and Complications in Pregnancy Front Public Health 2290. 